This document is the Accepted Manuscript version of a Published Work that appeared in final form in The Journal of Physical Chemistry C, copyright (C) American Chemical Society after peer review and technical editing by the publisher. To access the final edited and published work see 10.1021/acs.jpclett.8b00536.

\title{
Initial Stages in the Formation of Nickel Phosphides
}

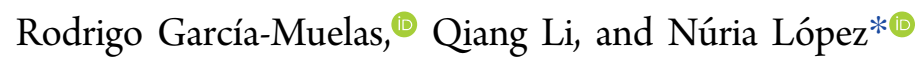 \\ Institute of Chemical Research of Catalonia, ICIQ The Barcelona Institute of Science and Technology, Av. Països Catalans, 16, 43007 \\ Tarragona, Spain
}

\begin{abstract}
Metal phosphides have emerged as a new powerful class of materials that can be employed as heterogeneous catalysts in transformations mainly to generate new energy vectors and the valorization of renewables. Synthetic protocols based on wet techniques are available and are based on the decomposition of the organic layer decorating the nanoparticles. For nickel, the phosphine of choice is trioctylphosphine, and this leads to the formation of $\mathrm{NiP}_{x}$ materials. However, the temperature at which the decomposition starts has been found to depend on the quality of the nickel surface. Density functional theory, DFT, holds the key to analyze the initial steps of the formation of these phosphide materials. We have found how clean nickel surfaces, either (111) or (100), readily breaks the ligand $\mathrm{P}-\mathrm{C}$ bonds. This triggers the

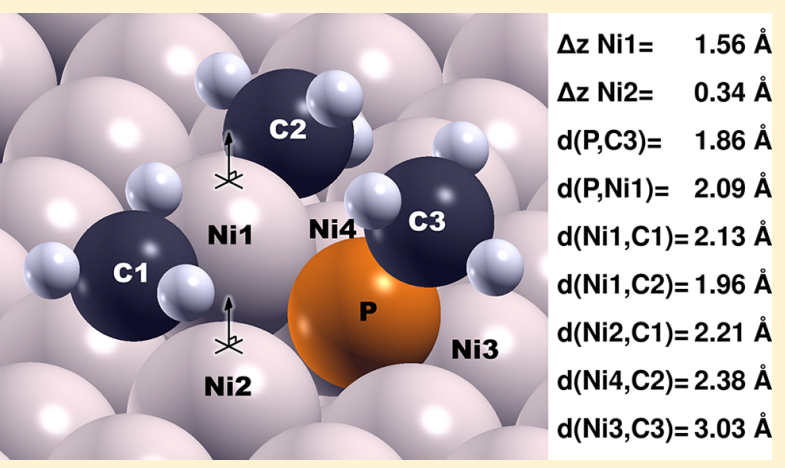
process that leads to the replacement of a surface nickel atom by $\mathrm{P}$ and concomintantly forms a Ni adatom on the surface surrounded by two methyl groups, thus starting the formation of the $\mathrm{NiP}_{x}$ phase. The whole process requires low energies, in agreement with the low temperature found in the experiments, $150{ }^{\circ} \mathrm{C}$. In contrast, if the surface is oxidized, the reaction does not proceed at low temperatures and oxygen vacancies need to be created first to start the $\mathrm{P}-\mathrm{C}$ bond breaking on the $\mathrm{Ni}$-clean patches. Our results show that the cleaner the surface is, the milder the reactions are required for the $\mathrm{NiP}_{x}$ formation, and thus they pave the way for gentler synthetic protocols that can improve the control of these materials.
\end{abstract}

\section{INTRODUCTION}

Metal phosphides are attracting a large amount of attention, as they have appealing properties in different areas of physics and chemistry. ${ }^{1}$ The applications extend from optics ${ }^{2,3}$ to magnets ${ }^{4,5}$ and for energy storage. ${ }^{6,7}$ Among these applications, one of the fastest growing areas is the use of metal phosphides as catalysts. Initially, they were used in hydrotreatment and selective hydrogenations, ${ }^{8-10}$ but nowadays, energy vector generation is the blooming area. ${ }^{11-13}$ Particularly, growing attention has been put in the hydrogen evolution reaction (HER) of the $\mathrm{Ni}_{2} \mathrm{P}$ and CoP compositions, ${ }^{14-23}$ but also on the $\mathrm{H}_{2}$ production from $\mathrm{BH}_{3} \mathrm{NH}_{3}{ }^{24,25}$ These properties appear as a consequence of the modifications in the ensemble, and the electronic structure of these materials. ${ }^{26}$ Thus, the $\mathrm{P}$ atoms in the lattice can be seen as embedded lattice poisons. In some cases, these materials present an almost continuum phase diagram with different $\mathrm{P}$ contents ${ }^{27,28}$ and thus some stability issues can be foreseen. The nature of the particular $\mathrm{Ni}-\mathrm{P}$ phase is highly relevant in the catalytic performance; for instance, different performances are found for the HER reaction on $\mathrm{Ni}_{2} \mathrm{P}$, $\mathrm{Ni}_{12} \mathrm{P}_{5}$, and $\mathrm{Ni}_{5} \mathrm{P}_{4}$, ${ }^{21}$ and thus, a strict control on the synthetic procedures that lead to the formation of the active phase is required.

Very recently, colloidal protocols to produce these materials have been generalized. The synthetic routes are based on the formation of nanoparticles decorated by phosphine ligands that evolve toward the formation of the phosphides by thermal treatment. For instance, the tri- $n$-octylphosphine, TOP, is employed both to decorate the nanoparticles and to control their size and as the source for $\mathrm{P}$. The reaction then is performed at medium temperatures of about $250{ }^{\circ} \mathrm{C}$ and pressures close to the normal, ambient pressure ${ }^{29,30}$ and can generate phosphides for a wide variety of reactive metals. For $\mathrm{Ni}$, two phases, $\mathrm{Ni}_{2} \mathrm{P}$ and $\mathrm{Ni}_{12} \mathrm{P}_{5}$, can be prepared by changing the conditions. ${ }^{31,32}$ The decomposition of the phosphide precursor depends on the metal, and for $\mathrm{Ni}$, the starting temperature for decomposition was reported to be $210{ }^{\circ} \mathrm{C},{ }^{33}$ while the complete decomposition and crystallization of the phosphide occurs at $230{ }^{\circ} \mathrm{C} .{ }^{34}$ In a deep XPS study, Salmeron et al. ${ }^{35}$ were able to describe more precisely how the wet synthesis of the Ni-based phosphide proceeds. In summary, when a Ni metal foil is clean, the surface can be decorated with TOP ligands that limit the growth of the nanoparticles. Upon heating to $150{ }^{\circ} \mathrm{C}$, the phosphines start degrading, and as a consequence, the $\mathrm{P}$ appears. However, this process is slightly more complex. Particularly, when the synthesis starts from nanoparticles, the decomposition of the organic fragment leads to the formation of a C-containing $\mathrm{Ni}$ core and the outermost surface is mostly $\mathrm{NiP}$ with $\mathrm{Ni}_{2} \mathrm{P}$ and $\mathrm{Ni}_{5} \mathrm{P}_{4}$ stoichiometries. 
Care shall be taken also, as $\mathrm{Ni}$ is relatively easy to oxidize. When starting by oxidized nanostructures, the phosphine decomposition starts only at $250{ }^{\circ} \mathrm{C}$. The process is schematically represented in Figure 1. Density functional

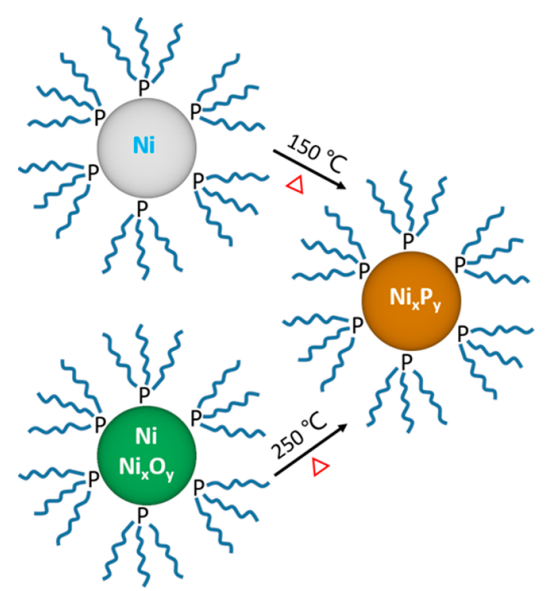

Figure 1. Required conditions for the formation of the NiP phase from $\mathrm{Ni}$. The different color codes show the dominating phases all throughout the manuscript (metallic $\mathrm{Ni}$ gray, oxidized green, phosphide orange). Adapted from Carenco et al. ${ }^{35}$

theory simulations have been extensively used to analyze the chemical properties of materials including phosphides. However, DFT is less often applied in the study of wet chemistry and synthetic protocols, although we have shown the potentiality of the approach in a wide series of systems. ${ }^{36-39}$ For instance, we have analyzed the role of surfactants when driving the structure of nanoparticles, controlling the catalytic properties therein. ${ }^{40-46}$ However, in all of the cases considered so far, the surfactants kept their entity under reaction conditions and the surface was poorly reactive. In the present work, we aim at investigating the evolution of the ligand on a relatively active surface as well as its decomposition and integration in the lattice as the first step in the formation of the phosphide phase.

\section{COMPUTATIONAL DETAILS}

The initial steps for the formation of phosphide under wet synthesis conditions were investigated by density functional theory, DFT, applied to slabs representing different nickel phases. The calculations were performed with the $\operatorname{VASP}^{47}$ code, and the functional of choice was PBE. ${ }^{48}$ Inner electrons were replaced by $\mathrm{PAW},{ }^{49}$ and the valence electrons were expanded in plane waves with a cutoff energy of $450 \mathrm{eV}$. The pure $\mathrm{Ni}(111)$ and (100) surfaces were described by a four-layer slab in a $p(3 \times 3)$ supercell with spin polarization; see Figure 2 . The $\mathrm{NiO}$ structure was constructed similarly: the slab contained four layers, and the $U$ parameter was set to $5.4 \mathrm{eV}$ following previous computational studies. ${ }^{50,51}$ The $k$-point were sampled by a $\Gamma$-centered $4 \times 4 \times 1$ Monkhorst-Pack ${ }^{52}$ grid. The adsorbates were only placed in one side of the slabs and interleaved by at least $14 \AA$. Dipole correction along the $z$ direction has been considered, ${ }^{53}$ and $\mathrm{vdW}$ interaction have been employed via the DFT-D3 method developed by Grimme et al. ${ }^{54}$ The large phosphines used in the experimental synthetic protocol were replaced by small surrogates $\mathrm{P}\left(\mathrm{CH}_{3}\right)_{3}$. Transition states were located with the climbing image version of the

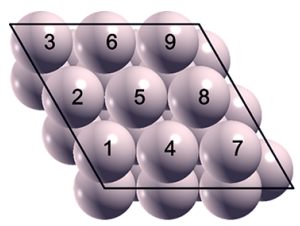

$\mathrm{Ni}(111)$

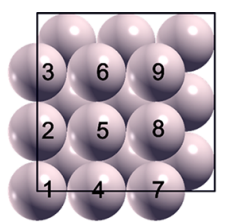

$\mathrm{Ni}(100)$

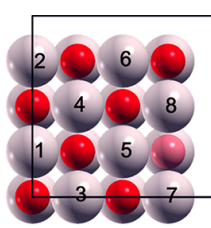

$\mathrm{NiO}(100)$
Figure 2. Top views of the models employed for the $\mathrm{Ni}(111)$ and (100) surfaces and for the oxide phase $\mathrm{NiO}(100)$ and the labels for the corresponding surface atoms. Gray $\mathrm{Ni}$, red $\mathrm{O}$, and fading $\mathrm{O}$ the position where the vacancy is formed.

nudged elastic band method. ${ }^{55}$ All of the structures can be retrieved from our ioChem-BD repository. ${ }^{56}$

\section{RESULTS}

Four model systems have been used to characterize the fosfine decomposition and initial steps of the formation of the phosphide phase. As the trioctylphosphine (TOP) employed in the experiments is very large, instead a surrogate compound trimethylphosphine $\mathrm{P}\left(\mathrm{CH}_{3}\right)_{3}$ (TMP) has been used as reacting phosphine. The replacement would have an effect provided that the packing or stabilization contributions with respect to the solvent were investigated, but for the purpose of analyzing the decomposition, the effect is minor, as the interactions are local in nature. Phosphine decomposition in the model systems has been summarized in Figure 3. The first model corresponds to a
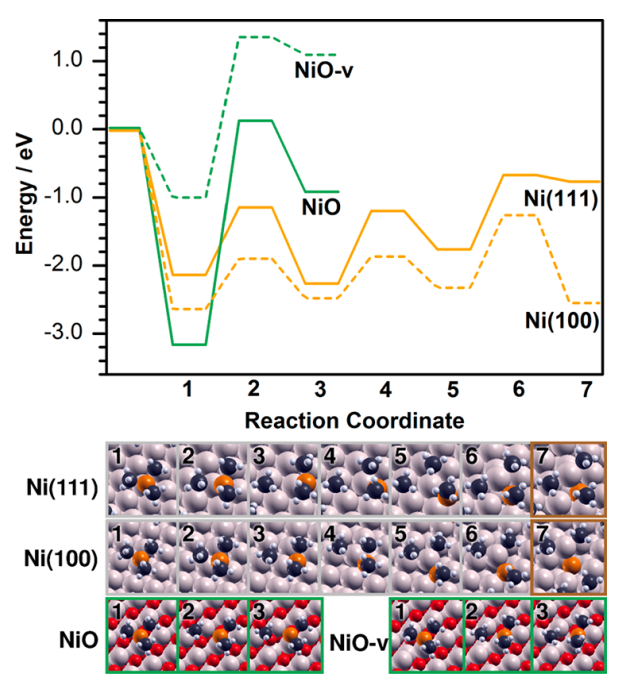

Figure 3. Reaction energy profile for the decomposition of $\mathrm{P}\left(\mathrm{CH}_{3}\right)_{3}$ on $\mathrm{Ni}(111)$ and $\mathrm{Ni}(100)$ (gray-orange) and $\mathrm{NiO}(100)$, both on the clean and oxygen-depleted surface (green continuous and dashed, respectively). The insets represent the most relevant structures along the reaction coordinates. Gray spheres stand for $\mathrm{Ni}$, red for $\mathrm{O}$, orange for $\mathrm{P}$, black for $\mathrm{C}$, and $\mathrm{H}$ are white.

pure $\mathrm{Ni}(111)$ surface that represents the clean surface model (dark gray); this is complemented by using the second lowest energy surface $\mathrm{Ni}(100)$ as the second model for which the reaction has also been inspected (gray). A third model represents a completely oxidized nickel system shown by the $\mathrm{NiO}(100)$ surface (green); this is the lowest energy surface for the oxide. The fourth model corresponds to a $\mathrm{NiO}(100)$ surface where an oxygen vacancy site has been created (green dashed), as oxides can lose oxygen atoms and they create 
Table 1. Magnetic Moments, $\mu_{\mathrm{B}}$, for the Most Relevant Ni Atoms during the Formation of the Phosphide Phase ${ }^{a}$

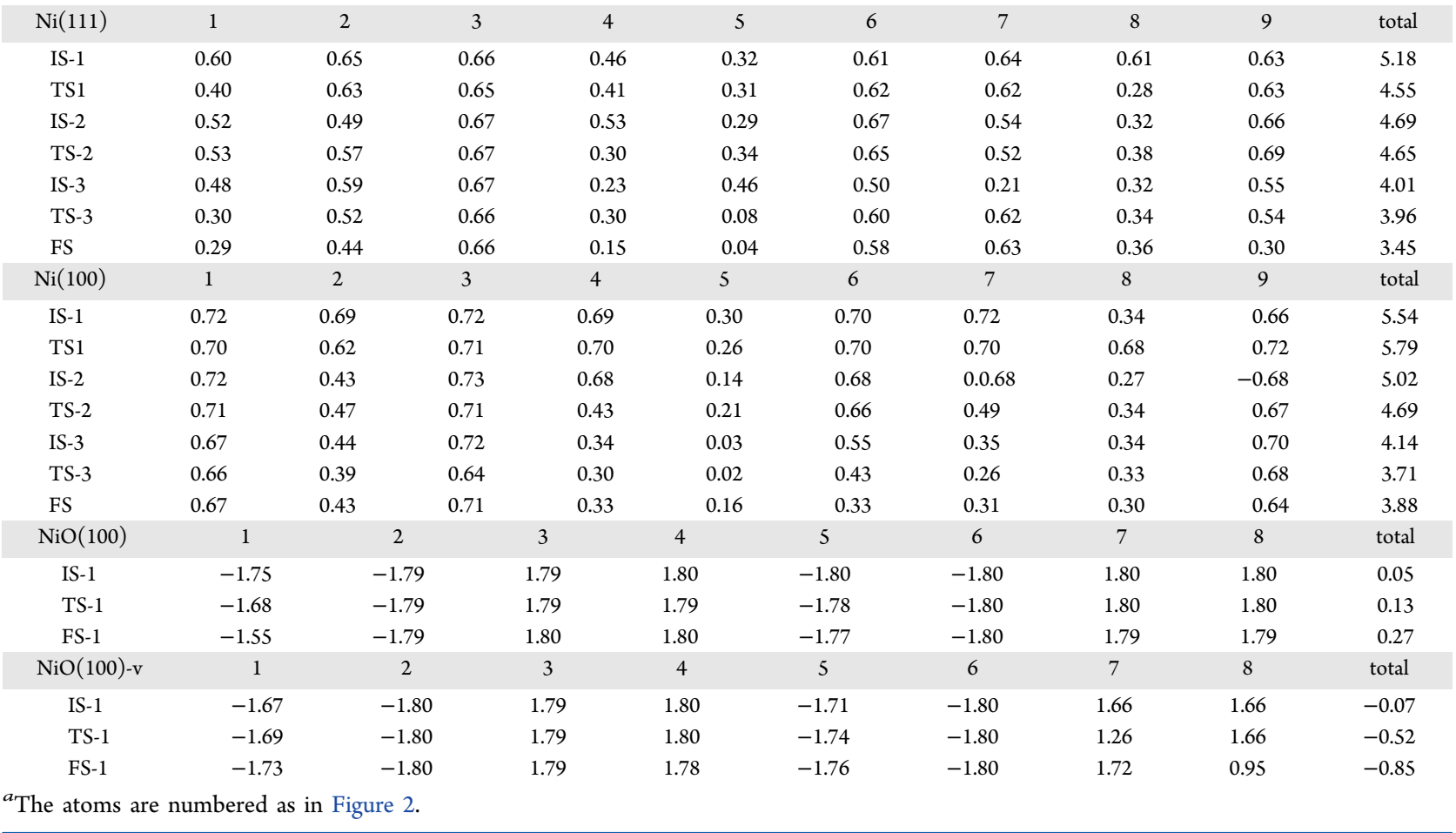

Table 2. Bader Charges, in $\mid \mathrm{e}^{-} \mathrm{l}$, for the Surface Ni Atoms during the Formation of the Phosphide Phase ${ }^{a}$

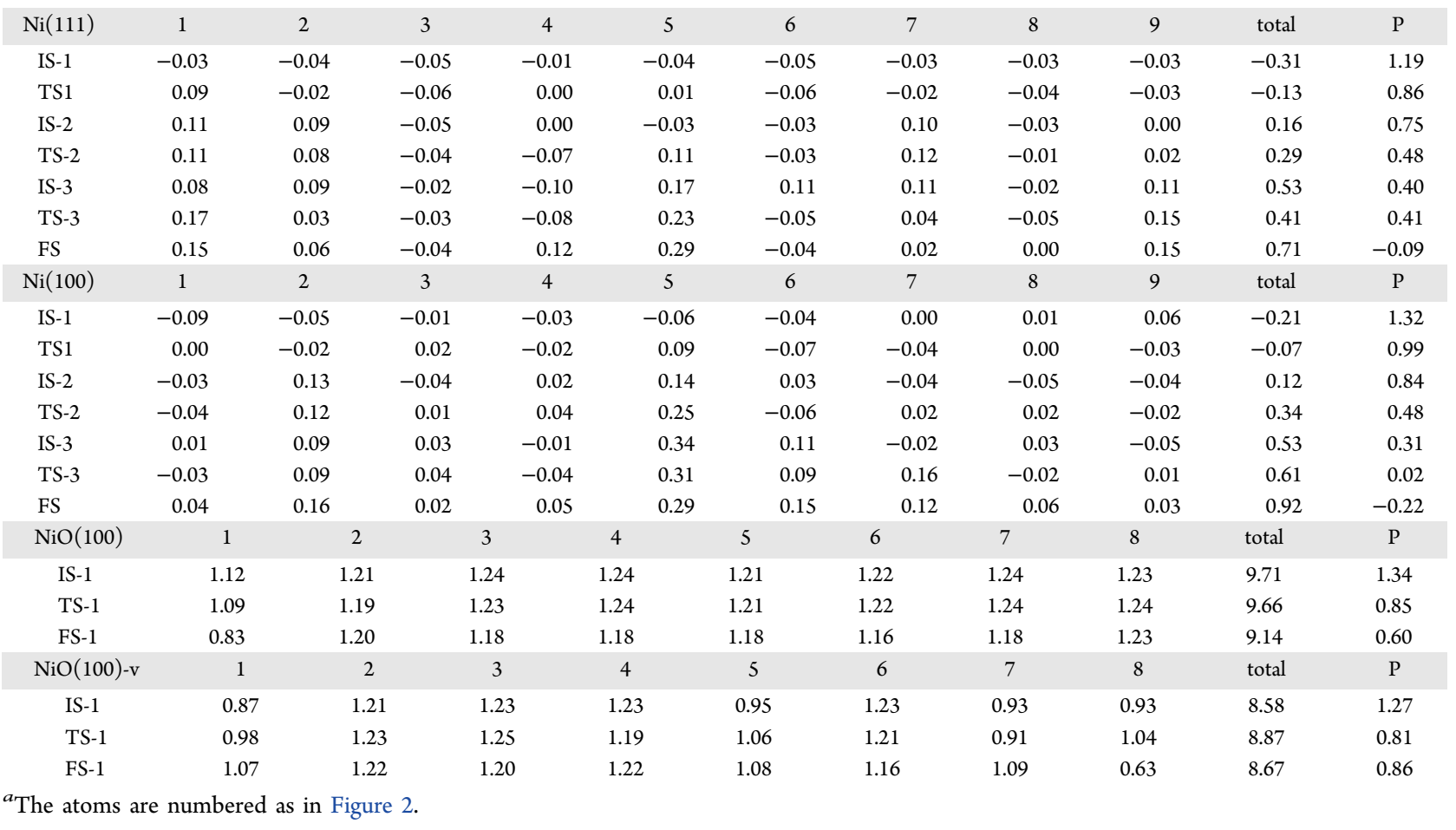

vacancies that present completely different properties when increasing the temperature. ${ }^{57}$ The magnetic moments and Bader charges ${ }^{58}$ of the surface $\mathrm{Ni}$ atoms and $\mathrm{P}$ are presented in Tables 1 and 2.

The phosphines adsorb on the most stable surface termination of $\mathrm{Ni}(111)$ by $-2.14 \mathrm{eV}$, with the $\mathrm{P}$ atom directly in contact with one $\mathrm{Ni}$ and effectively quenching the magnetic moment of the $\mathrm{Ni}$ atom to which it is directly adsorbed due to the donor nature of the lone pair from the $\mathrm{P}$ atom. From this starting point, the phosphine can break; see Figure 3. The first $\mathrm{P}-\mathrm{C}$ breaking shows a rather small barrier of about $0.99 \mathrm{eV}$ and results in a configuration where the methyl group migrates to 

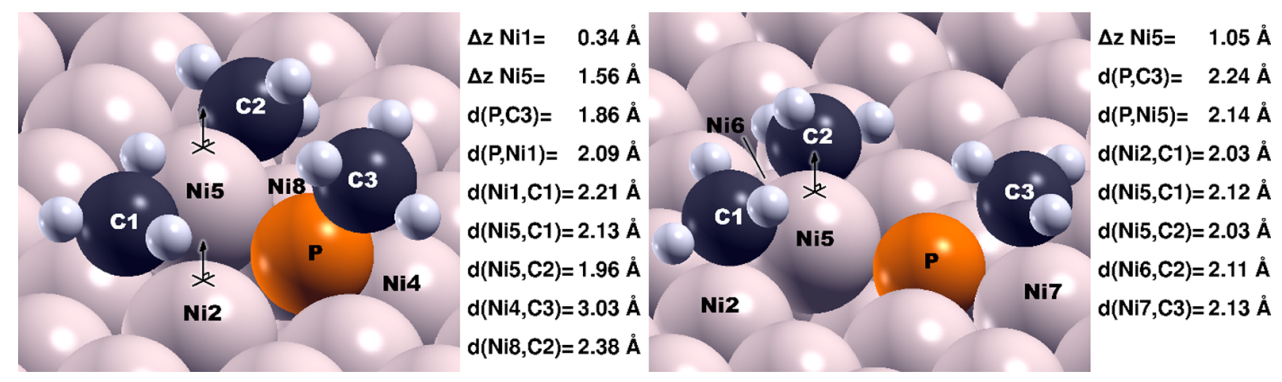

Figure 4. Transition state structures (TS-3) leading to the formation of the phosphide with the most relevant distances for the first Ni replacement by P (left (111) surface, right (100) surface). The origin of the stabilization of adatoms by the methyl groups is highlighted. Gray spheres stand for $\mathrm{Ni}$, orange for $\mathrm{P}$, black for $\mathrm{C}$, and white for $\mathrm{H}$. The atoms are numbered as in Figure 2 .

the surface and the remaining $\mathrm{P}\left(\mathrm{CH}_{3}\right)_{2}$ fragment changes from a top to a bridge-like configuration to satisfy the valence of $\mathrm{P}$. The overall reaction is slightly exothermic. This finding agrees with the experimental observations on $\mathrm{Ni}$ nanoparticles or organometallic compounds with phosphine ligands for which bond cleavage was observed at temperatures about $100^{\circ} \mathrm{C}^{59,60}$ The elimination of the second methyl group requires a slightly larger energy barrier of $1.07 \mathrm{eV}$. In that case, the overall thermodynamic cost for this step is endothermic by $0.51 \mathrm{eV}$. In this configuration, one of the $\mathrm{Ni}$ atoms from the surface is already displaced out of the equilibrium position, by $0.16 \AA$, and thus points toward the formation of an adatom. ${ }^{61}$ The dimethyl coordinated phosphine is then reactive enough to start penetration into the lattice. The final step leads to the bond breaking of the third $\mathrm{P}-\mathrm{C}$ bond and the full replacement of one of the $\mathrm{Ni}$ atoms from the surface by the $\mathrm{P}$, thus becoming the seed of the nickel phosphide formation. The reaction is uphill by $1 \mathrm{eV}$, and the activation barrier is $1.09 \mathrm{eV}$. In the final structure, one of the methyl groups sits on the $\mathrm{Ni}$ surface and the total magnetic moment is reduced due to the $\mathrm{Ni}-\mathrm{C}$ bond formation, from the starting value of $5.18 \mu_{\mathrm{B}}$ for the surface layer down to 4.69 , then 4.01 after one and two methyl cleaves, and $3.45 \mu_{\mathrm{B}}$ for the final structure. In parallel, the initial positive charge of $\mathrm{P}$, about $1.2 \mathrm{le}^{-l}$, gets reduced down to the final state where it is slightly negatively charged, -0.1 , while the metal surface changes from slightly negative to positive $0.7 \mathrm{le}^{-} \mathrm{l}$. In the final configuration, detailed in Figure 4, the adatom is coordinated to the two methyl groups (distances 2.13 and $1.96 \AA$ and the $\mathrm{Ni}$ atom is extracted from the plane by $2.40 \AA$ ). The energy cost of forming the adatom (roughly $2 / 3$ of the cohesive energy) is counterbalanced by the increasing interaction with the methyl fragments and $\mathrm{PCH}_{3}$, which also moves toward a fcc-like configuration on the surface to saturate as many bonds as possible. The overall energy profile starting from the adsorbed phosphine is $1.37 \mathrm{eV}$ uphill with the largest barrier being $1.47 \mathrm{eV}$, which agrees very well with the temperature of $150{ }^{\circ} \mathrm{C}$ reported in the experiments needed to see the initial spectroscopic features corresponding to the formation of the nickel phosphide phase. Further adsorption of the phosphine on the adatom is much less exothermic, around $0.7 \mathrm{eV}$. Therefore, scavenging atoms from another area of the clean surface would be more favorable than reacting on the formed adatom.

Alternatively, the second most stable surface (100) has been set as the initial state. The coverage considered for the phosphine is the same as that in the previous case. Initially, the phosphine adsorbs to a single $\mathrm{Ni}$ atom on the surface, and the binding energy is slightly larger than that for the (111) surface,
$-2.36 \mathrm{eV}$. The reaction proceeds in the same manner as described for the (111) surface, with the barriers for the different $\mathrm{P}-\mathrm{CH}_{3}$ cleavages being $0.74,0.75$, and $0.74 \mathrm{eV}$. The overall process is more exothermic than that on (111), and the barriers are smaller, since the coordination number of the (100) surface is smaller than that of the (111), which allows larger reorganizations at a lower energy cost. The magnetic moments behave similarly to the (111) case, as from an initial magnetization of $5.54 \mu_{\mathrm{B}}$, the value decreases to $5.02,4.14$, and $3.88 \mu_{\mathrm{B}}$ during the different cleavages. The corresponding charges for $\mathrm{P}$ are positive by $1.3 \mathrm{le}^{-} \mathrm{I}$ in the starting configuration and $-0.2 \mathrm{le}^{-} \mathrm{l}$ in the final state, while the surface goes from slightly negative, -0.2 , to $0.9 \mathrm{le}^{-} \mathrm{l}$ in the surface layer. Again, as for the (111) surfaces, the last step ends with the replacement of the surface $\mathrm{Ni}$ by a $\mathrm{P}$ atom and the formation of the adatom stabilized by the two methyl groups. The last transition states where the replacement occurs are represented in Figure 4, and show that the $\mathrm{Ni}$ atom is less displaced upward in the (100) surface because the surface is less compact than the (111).

On the nickel oxide, TMP adsorption is very exothermic, $-3.16 \mathrm{eV}$, and takes place on top of the $\mathrm{Ni}$ cations. The dissociation could only occur heterolytically, as the activation in many oxides, ${ }^{62}$ and ends up forming a cationic $\mathrm{CH}_{3}{ }^{+}$fragment that is attached to a lattice oxygen and the negatively charged $\mathrm{P}\left(\mathrm{CH}_{3}\right)_{2}$ still bonded to the cation. Although the phosphine adsorption is very exothermic, the dissociation is very endothermic, about $2.24 \mathrm{eV}$. This is due to two fundamental terms: (i) the $\mathrm{C}-\mathrm{O}$ bond formation is not very effective and (ii) the phosphine cannot coordinate properly to two cations on the surface, as they are far apart in the structure. In the initial structure, the $\mathrm{P}$ is positively charged, $1.3 \mathrm{le}^{-} \mathrm{l}$, and this charge is reduced along the reaction path to $0.6 \mathrm{le}^{-} \mathrm{l}$, which is compensated by the charge variation in one of the $\mathrm{Ni}$ atoms that also reduces its magnetization. The consequence is that the fully oxidized $\mathrm{NiO}$ surface is inert with respect to phosphines and then an alternative mechanism needs to be identified. As oxides are known to lose oxygen when the temperature is raised, we included a model showing a surface oxygen vacancy. In the case of $\mathrm{NiO}$, the vacancy formation requires $2.32 \mathrm{eV}$. On the model system close to a surface oxygen vacancy, the adsorption of the basic $\mathrm{P}\left(\mathrm{CH}_{3}\right)_{3}$ is less favorable than $\mathrm{Ni}$ or $\mathrm{NiO}$. The first step in the demethylation is now endothermic by $2.10 \mathrm{eV}$, and the barrier is $2.35 \mathrm{eV}$. Therefore, the process is also unlikely on this surface under the reaction conditions described in the experiments. The interpretation of the results is thus clear: the NiO surface can trap the surfactant but would require extensive reduction with the formation of $\mathrm{Ni}$ patches 
before the reaction occurs. In summary, our results are compatible with the $250{ }^{\circ} \mathrm{C}$ temperature needed to start the phosphine decomposition effectively on the Ni-only patches of the surface of the oxides that are already oxygen depleted. As we have indicated in the Introduction, our calculations only represent the very first stages of the formation of the NiP phase. The methyl groups on the surface could decompose further following the paths that have been already described in the literature. $^{63}$

\section{DISCUSSION}

For the metal $\mathrm{Ni}(111)$ and (100) surfaces, the barriers for all of the steps follow two Brønsted-Evans-Polanyi ${ }^{64}$ relationships (see Figure 5); this indicates the local nature of the bond that

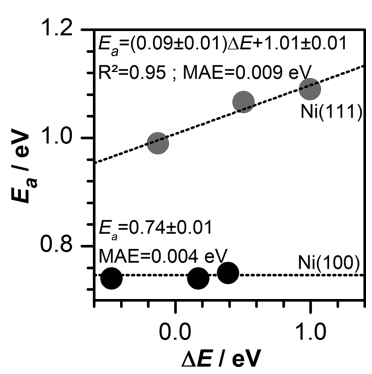

Figure 5. Brønsted-Evans-Polanyi (BEP) relationship between the activation energy for each of the $\mathrm{P}-\mathrm{C}$ steps as a function of the reaction energy for each particular step on the $\mathrm{Ni}(111)$ and $\mathrm{Ni}(100)$ surfaces. The numbers correspond to the transition state structures identified in Figure 3. Notice that $\mathrm{NiO}(100)$ surface would not follow the same BEP, as the bond-breaking transition state corresponds to a heterolytic path.

drives the formation of the adatom and the phosphide structure. The reason for the lower values found for $\mathrm{Ni}(100)$ can be traced back to the more open nature of the surface that allows lower energy processes and is slightly more flexible. Therefore, this would allow one to couple the present results to the coordination dependence rules to expand the thermodynamic and kinetic requirements to finite size nanoparticles and low coordinated sites. ${ }^{65}$ However, it shall be noticed that the low barriers found for all of the steps imply that the activation can easily occur even in the denser packed surfaces; thus, no important size contributions are expected.

The present replacement mechanism is related to several other observations in the literature. For instance, in homogeneous catalysis, the reductive cleavage of $\mathrm{P}-\mathrm{C}$ bonds and the concomitant formation of phosphido species is extensively reported in the literature for low-valent metal complexes. ${ }^{66}$ The formation of adatoms in reactive environments has been identified in other contexts, like the adsorption of S-containing thiols on gold, ${ }^{67}$ or under reaction conditions by reaction-deposition-elimination cycles ${ }^{68}$ or the identification of organometallic-like compounds as intermediates on surface $\mathrm{C}-\mathrm{C}$ couplings. ${ }^{61}$

\section{CONCLUSIONS}

Density functional theory simulations have been employed to unravel the initial states of the decomposition of ligands based on phosphines to form metal phosphides. We have found that the reaction is slightly endothermic on the bare $\mathrm{Ni}(111)$ and (100) surfaces, but it proceeds smoothly with relatively small energy barriers. The values obtained are compatible with the temperatures at which the experiments are carried out. In addition, the reaction paths encompass the extraction of $\mathrm{Ni}$ atoms from the surface and their replacement by the reactive $\mathrm{P}$ from the ligand. However, nickel reacts easily with oxygen; therefore, many surfaces are better described as oxides. On $\mathrm{Ni}$ oxides the phosphidation reaction is very endothermic and thus cannot proceed. Even when they are partially oxygen depleted, oxide surfaces are not reactive enough to allow the decomposition of the phosphine. Therefore, the reaction can only proceed by increasing the overall temperature and will take place on the Ni patches of the surface. The number of potential simultaneous paths leading to full decomposition and phosphide formation calls for an integrated effort where detailed characterization with in situ techniques can be coupled to density functional theory models to fully describe the reaction mechanisms involved. The present results open a new path toward the rational understanding of synthetic protocols and pave the way for establishing new, more suitable ligands that can allow milder reaction conditions and thus more control on the synthesis.

\section{AUTHOR INFORMATION}

\section{Corresponding Author}

*E-mail: nlopez@iciq.es.

\section{ORCID}

Rodrigo García-Muelas: 0000-0002-2219-5027

Núria López: 0000-0001-9150-5941

\section{Notes}

The authors declare no competing financial interest.

Data-set collection of computational results, including all the structures, are available in: http://dx.doi.org/10.19061/ iochem-bd-1-42.

\section{ACKNOWLEDGMENTS}

The authors thank MINECO CTQ2015-68770-R project for financial support. The authors gratefully acknowledge the generous computing time and assistance provided by the Barcelona Supercomputing Center and the Spanish Supercomputing Network.

\section{REFERENCES}

(1) Carenco, S.; Portehault, D.; Boissiere, C.; Mezailles, N.; Sánchez, C. Nanoscaled Metal Borides and Phosphides: Recent Developments and Perspectives. Chem. Rev. 2013, 113, 7981-8065.

(2) Li, L.; Reiss, P. One-pot Synthesis of Highly Luminescent InP/ ZnS Nanocrystals without Precursor Injection. J. Am. Chem. Soc. 2008, 130, 11588-11589.

(3) Baek, J.; Allen, P. M.; Bawendi, M. G.; Jensen, K. F. Investigation of Indium Phosphide Nanocrystal Synthesis Using a High-Temperature and High-Pressure Continuous Flow Microreactor. Angew. Chem., Int. Ed. 2011, 50, 627-656.

(4) Booth, R. A.; Marinescu, M.; Liu, J.; Majetich, S. A. Preferential Crystallographic Alignment in Polycrystalline MnP. J. Magn. Magn. Mater. 2010, 322, 2571-2574.

(5) Carenco, S.; Le Goff, X. F.; Shi, J.; Roiban, L.; Ersen, O.; Boissiere, C.; Sanchez, C.; Mézailles, N. Magnetic Core-Shell Nanoparticles from Nanoscale-Induced Phase Segregation. Chem. Mater. 2011, 23, 2270.

(6) Pralong, V.; Souza, D. C. S.; Leung, K. T.; Nazar, L. F. Reversible Lithium Uptake by $\mathrm{CoP}_{3}$ at Low Potential: Role of the Anion. Electrochem. Commun. 2002, 4, 516-520.

(7) Carenco, S.; Surcin, C.; Morcrette, M.; Larcher, D.; Mézailles, N.; Boissière, C.; Sanchez, C. Improving the Li-Electrochemical Properties 
of Monodisperse $\mathrm{Ni}_{2} \mathrm{P}$ Nanoparticles by Self-Generated Carbon Coating. Chem. Mater. 2012, 24, 688-697.

(8) Alvarez-Galvan, M. C.; Blanco Brieva, G.; Sánchez, M. C.; Fierro, J. L. G. Metal Phosphide Catalysts for the Hydrotreatment of Nonedible Vegetable Oils. Catal. Today 2017, DOI: 10.1016/j.cattod.2017.03.031.

(9) Sweeny, N. P.; Rohrer, C. S.; Brown, O. W. Dinickel Phosphide as a Heterogeneous Catalyst for the Vapor Phase Reduction of Nitrobenzene with Hydrogen to Aniline and Water. J. Am. Chem. Soc. 1958, 80, 799-800.

(10) Layan Savithra, G. H.; Muthuswamy, E.; Bowker, R. H.; Carrillo, B. A.; Bussell, M. E.; Brock, S. L. Rational Design of Nickel Phosphide Hydrodesulfurization Catalysts: Controlling Particle Size and Preventing Sintering. Chem. Mater. 2013, 25, 825-833.

(11) Oyama, S. T.; Gott, T.; Zhao, H.; Lee, Y.-K. Transition Metal Phosphide Hydroprocessing Catalysts: A Review. Catal. Today 2009, 143, 94-107.

(12) Carenco, S.; Leyva-Perez, A.; Concepcion, P.; Boissiere, C.; Mezailles, N.; Sanchez, C.; Corma, A. Nickel Phosphide Nanocatalysts for the Chemoselective Hydrogenation of Alkynes. Nano Today 2012, 7, 21-28.

(13) Prins, R.; Bussell, M. E. Metal Phosphides: Preparation, Characterization and Catalytic Reactivity. Catal. Lett. 2012, 142, 1413-1436.

(14) Popczun, E. J.; McKone, J. R.; Read, C. G.; Biacchi, A. J.; Wiltrout, A. M.; Lewis, N. S.; Schaak, R. E. Nanostructured Nickel Phosphide as an Electrocatalyst for the Hydrogen Evolution Reaction. J. Am. Chem. Soc. 2013, 135, 9267-9270.

(15) Pu, Z.; Liu, Q.; Tang, C.; Asiri, A. M.; Sun, X. $\mathrm{Ni}_{2}$ P Nanoparticle Films Supported on a Ti Plate as An Efficient Hydrogen Evolution Cathode. Nanoscale 2014, 6, 11031-11034.

(16) Feng, L.; Vrubel, H.; Bensimon, M.; Hu, X. Easily-prepared Dinickel Phosphide $\left(\mathrm{Ni}_{2} \mathrm{P}\right)$ Nanoparticles as an Efficient and Robust Electrocatalyst for Hydrogen Evolution. Phys. Chem. Chem. Phys. 2014, 16, 5917.

(17) Popczun, E.; Read, C. G.; Roske, C. W.; Lewis, N. S.; Schaak, R. E. Highly Active Electrocatalysis of the Hydrogen Evolution Reaction by Cobalt Phosphide Nanoparticles. Angew. Chem. 2014, 126, 55315534.

(18) Stern, L. A.; Feng, L.; Song, F.; Hu, X. $\mathrm{Ni}_{2} \mathrm{P}$ As a Janus Catalyst for Water Splitting: the Oxygen Evolution Activity of $\mathrm{N}_{\mathrm{i} 2} \mathrm{P}$ Nanoparticles. Energy Environ. Sci. 2015, 8, 2347-2351.

(19) Cai, Z.; Song, X.; Wang, Y.; Chen, X. Electrodeposition-Assisted Synthesis of $\mathrm{Ni}_{2} \mathrm{P}$ Nanosheets on 3D Graphene/Ni Foam Electrode and Its Performance for Electrocatalytic Hydrogen Production. ChemElectroChem 2015, 2, 1665-1671.

(20) Shi, Y.; Zhang, B. Recent Advances in Transition Metal Phosphide Nanomaterials: Synthesis and Applications in Hydrogen Evolution Reaction. Chem. Soc. Rev. 2016, 45, 1529-1541.

(21) You, B.; Jiang, N.; Sheng, M.; Bushan, M. W.; Sun, Y. Hierarchically Porous Urchin-Like $\mathrm{Ni}_{2} \mathrm{P}$ Superstructures Supported on Nickel Foam as Efficient Bifunctional Electrocatalysts for Overall Water Splitting. ACS Catal. 2016, 6, 714-721.

(22) Xiao, P.; Chen, W.; Wang, X. A Review of Phosphide-Based Materials for Electrocatalytic Hydrogen Evolution. Adv. Energy Mater. 2015, 5, 1500985.

(23) Callejas, J. F.; Read, C. G.; Roske, C. W.; Lewis, N. S.; Schaak, R. E. Synthesis, Characterization, and Properties of Metal Phosphide Catalysts for the Hydrogen-Evolution Reaction. Chem. Mater. 2016, 28, 6017-6044.

(24) Peng, C.-Y.; Knag, L.; Shuang, C.; Chen, Y.; Lin, Z.-S.; Fu, W.-F. Nanostructured $\mathrm{Ni}_{2} \mathrm{P}$ as a Robust Catalyst for the Hydrolytic Dehydrogenation of Ammonia-Borane. Angew. Chem., Int. Ed. 2015, 54, 15725-15729.

(25) Hou, C.-C.; Li, Q.; Peng, C.-J.; Chen, Q.-Q.; Ye, H.-F.; Fu, W.F.; Che, C.-M.; Lopez, N.; Chen, Y. Ternary Ni-Co-P Nanoparticles as Noble-metal-free Catalysts to Boost the Hydrolytic Dehydrogenation of Ammonia-Borane. Energy Environ. Sci. 2017, 10, 1770-1776.
(26) Hansen, M. H.; Stern, L.-A.; Feng, L.; Rossmeisl, J.; Hu, X. Widely Available Active Sites on $\mathrm{Ni}_{2} \mathrm{P}$ for Electrochemical Hydrogen Evolution - Insights from First Principles Calculations. Phys. Chem. Chem. Phys. 2015, 17, 10823-10829.

(27) http://resource.npl.co.uk/mtdata/phdiagrams/nip.htm, accessed June 2017.

(28) Kucernak, A. R. J.; Sundaram, V. N. N. Nickel Phosphide: the Effect of Phosphorus Content on Hydrogen Evolution Activity and Corrosion Resistance in Acidic Medium. J. Mater. Chem. A 2014, 2, 17435-17445.

(29) Park, J. G. J.; Koo, B.; Yoon, K. Y.; Hwang, Y.; Kang, M.; Hyeon, T. Generalized Synthesis of Metal Phosphide Nanorods via Thermal Decomposition of Continuously Delivered Metal-Phosphine Complexes Using a Syringe Pump. J. Am. Chem. Soc. 2005, 127, 84338440 .

(30) Wang, J.; Johnston-Peck, A. C.; Tracy, J. B. Nickel Phosphide Nanoparticles with Hollow, Solid, and Amorphous Structures. Chem. Mater. 2009, 21, 4462-4467.

(31) Muthuswamy, E.; Savithra, G. H. L.; Brock, S. L. Synthetic Levers Enabling Independent Control of Phase, Size, and Morphology in Nickel Phosphide Nanoparticles. ACS Nano 2011, 5, 2402-2411.

(32) Muthuswamy, E.; Brock, S. L. Solid-state Phase Transformations in Solution: Templated Conversion of Nanoscale Nickel Phosphides. Chem. Commun. 2011, 47, 12334-12336.

(33) Moreau, L. M.; Ha, D.-H.; Bealing, C. R.; Zhang, H.; Henning, R. G.; Robinson, R. D. Unintended Phosphorus Doping of Nickel Nanoparticles During Synthesis with TOP: A Discovery Through Structural Analysis. Nano Lett. 2012, 12, 4530-4539.

(34) Moreau, L. M.; Ha, D.-H.; Zhang, H.; Hovden, R.; Muller, D. A.; Robison, R. D. Defining Crystalline/Amorphous Phases of Nanoparticles Through X-ray Absorption Spectroscopy and X-ray Diffraction: The Case of Nickel Phosphide. Chem. Mater. 2013, 25, 2394-2403.

(35) Carenco, S.; Liu, Z.; Salmeron, M. The Birth of Nickel Phosphide Catalysts: Monitoring Phosphorus Insertion into Nickel. ChemCatChem 2017, 9, 2318-2323.

(36) Heinz, H.; Pramanik, C.; Heinz, O.; Ding, Y.; Mishra, R. K.; Marchon, D.; Flatt, R. J.; Estrela-Lopis, I.; Llope, J.; Moya, S.; Ziolo, R. F. Nanoparticle Decoration with Surfactants: Molecular Interactions, Assembly, and Applications. Surf. Sci. Rep. 2017, 72, 1-58.

(37) Barmparis, G. D.; Lodziana, Z.; Lopez, N.; Remediakis, I. N.; Beilstein, J. Nanoparticle Shapes by Using Wulff Constructions and First-principles Calculations. Beilstein J. Nanotechnol. 2015, 6, 361368.

(38) Gomez-Graña, S.; Goris, B.; Altantzis, T.; Fernandez-Lopez, C.; Carbo-Argibay, E.; Guerrero-Martinez, A.; Almora-Barrios, N.; Lopez, N.; Pastoriza-Santos, I.; Pérez-Juste, J.; Bals, S.; van Tendeloo, G.; LizMarzan, L. M. Au@Ag Nanoparticles: Halides Stabilize $\{100\}$ Facets. J. Phys. Chem. Lett. 2013, 4, 2209-2216.

(39) Almora-Barrios, N.; Novell-Leruth, G.; Whiting, P.; Liz-Marzan, L. M.; López, N. Theoretical Description of the Role of Halides, Silver, and Surfactants on the Structure of Gold Nanorods. Nano Lett. 2014, 14, 871-875.

(40) Vile, G.; Almora-Barrios, N.; Mitchell, S.; López, N.; PérezRamírez, J. From the Lindlar Catalyst to Supported Ligand-Modified Palladium Nanoparticles: Selectivity Patterns and Accessibility Constraints in the Continuous-Flow Three-Phase Hydrogenation of Acetylenic Compounds. Chem. - Eur. J. 2014, 20, 5926-5937.

(41) Albani, D.; Vile, G.; Mitchell, S.; Witte, P. T.; Almora-Barrios, N.; López, N.; Pérez-Ramírez, J. Ligand Ordering Determines the Catalytic Response of Hybrid Palladium Nanoparticles in Hydrogenation. Catal. Sci. Technol. 2016, 6, 1621-1631.

(42) Albani, D.; Li, Q.; Vile, G.; Mitchell, S.; Almora-Barrios, N.; López, N.; Pérez-Ramírez, J. Interfacial Acidity in Ligand-modified Ruthenium Nanoparticles Boosts the Hydrogenation of Levulinic Acid to Gamma-valerolactone. Green Chem. 2017, 19, 2361-2370.

(43) Almora-Barrios, N.; Vile, G.; Garcia-Ratés, M.; Pérez-Ramírez, J.; López, N. Electrochemical Effects at Surfactant-Platinum Nano- 
particle Interfaces Boost Catalytic Performance. ChemCatChem 2017, 9, 604-609.

(44) Lari, G. M.; Puertolas, B.; Shahrokhi, M.; López, N.; PérezRamírez, J. Hybrid Palladium Nanoparticles for Direct Hydrogen Peroxide Synthesis: The Key Role of the Ligand. Angew. Chem., Int. Ed. 2017, 56, 1775-1779.

(45) Almora-Barrios, N.; Cano, I.; van Leeuwen, P. W. N. M.; López, N. Concerted Chemoselective Hydrogenation of Acrolein on Secondary Phosphine Oxide Decorated Gold Nanoparticles. ACS Catal. 2017, 7, 3949-3954.

(46) Fiorio, J. L.; López, N.; Rossi, L. Gold-Ligand-Catalyzed Selective Hydrogenation of Alkynes into cis-Alkenes via $\mathrm{H}_{2}$ Heterolytic Activation by Frustrated Lewis Pairs. ACS Catal. 2017, 7, 2973-2980.

(47) Kresse, G.; Furthmüller, J. Efficiency of ab-initio Total Energy Calculations for Metals and Semiconductors Using a Plane-Wave Basis Set. Comput. Mater. Sci. 1996, 6, 15-50.

(48) Perdew, J. P.; Burke, K.; Ernzerhof, M. Generalized Gradient Approximation Made Simple. Phys. Rev. Lett. 1996, 77, 3865.

(49) Kresse, G.; Joubert, D. From Ultrasoft Pseudopotentials to the Projector Augmented-Wave Method. Phys. Rev. B: Condens. Matter Mater. Phys. 1999, 59, 1758.

(50) Wang, L.; Maxisch, T.; Ceder, G. Oxidation Energies of Transition Metal Oxides within the GGA+U Framework. Phys. Rev. B: Condens. Matter Mater. Phys. 2006, 73, 195107.

(51) Capdevila-Cortada, M.; Lodziana, Z.; López, N. Performance of $\mathrm{DFT}+\mathrm{U}$ Approaches in the Study of Catalytic Materials. ACS Catal. 2016, 6, 8370-8379.

(52) Monkhorst, H. J.; Pack, J. D. Special Points for Brillouin-zone Integrations. Phys. Rev. B 1976, 13, 5188.

(53) Makov, G.; Payne, M. C. Periodic Boundary Conditions in $a b$ initio Calculations. Phys. Rev. B: Condens. Matter Mater. Phys. 1995, 51, 4014-4022.

(54) Grimme, S.; Antony, J.; Ehrlich, S.; Krieg, S. A Consistent and Accurate $a b$ initio Parametrization of Density Functional Dispersion Correction (DFT-D) for the 94 Elements H-Pu. J. Chem. Phys. 2010, 132, 154104

(55) Henkelman, G.; Jónsson, H. Improved Tangent Estimate in the Nudged Elastic Band Method for Finding Minimum Energy Paths and Saddle Points. J. Chem. Phys. 2000, 113, 9978-9985.

(56) Alvarez-Moreno, M.; De Graaf, C.; López, N.; Maseras, F.; Poblet, J. M.; Bo, C. Managing the Computational Chemistry Big Data Problem: The ioChem-BD Platform. J. Chem. Inf. Model. 2015, 55, 95-103.

(57) Carrasco, J.; López, N.; Illas, F. First Principles Analysis of the Stability and Diffusion of Oxygen Vacancies in Metal Oxides. Phys. Rev. Lett. 2004, 93, 225502.

(58) Henkelman, G.; Arnaldsson, A.; Jónsson, H. A Fast and Robust Algorithm for Bader Decomposition of Charge Density. Comput. Mater. Sci. 2006, 36, 354.

(59) Garrou, P. E. Transition-Metal-Mediated Phosphorus-Carbon Bond Cleavage and Its Relevance to Homogeneous Catalyst Deactivation. Chem. Rev. 1985, 85, 171-185.

(60) Acosta-Ramírez, A.; Flores Alamo, M.; Jones, W. D.; Garcia, J. J. $\mathrm{P}-\mathrm{C}$ Bond Scission at the TRIPHOS Ligand and $\mathrm{C}-\mathrm{CN}$ Bond Cleavage in 2-Methyl-3-butenenitrile with $\left[\mathrm{Ni}(\mathrm{COD})_{2}\right]$. Organometallics 2008, 27, 1834-1840.

(61) Barton, D.; Gao, H.-Y.; Held, P. A.; Studer, A.; Doltsinis, N. L.; Neugerbauer, J. Formation of Organometallic Intermediate States in On-Surface Ullmann Couplings. Chem. - Eur. J. 2017, 23, 6190-6197.

(62) Garcia-Melchor, M.; López, N. Homolytic Products from Heterolytic Paths in $\mathrm{H}_{2}$ Dissociation on Metal Oxides: The Example of $\mathrm{CeO}_{2}$. J. Phys. Chem. C 2014, 118, 10921-10926.

(63) Jones, G.; Jakobsen, J. G.; Shim, S. S.; Kleis, J.; Andersson, M. P.; Rossmeisl, J.; Abild-Pedersen, F.; Bligaard, T.; Helveg, S.; Hinnemann, B.; et al. First Principles Calculations and Experimental Insight into Methane Steam Reforming over Transition Metal Catalysts. J. Catal. 2008, 259, 147-160.
(64) Evans, M. G.; Polanyi, M. Further Considerations on the Thermodynamics of Chemical Equilibria and Reaction Rates. Trans. Faraday Soc. 1936, 32, 1333.

(65) Calle-Vallejo, F.; Loffreda, D.; Koper, M. T. M.; Sautet, P. Introducing Structural Sensitivity into Adsorption-energy Scaling Relations by Means of Coordination Numbers. Nat. Chem. 2015, 7, 403-410.

(66) van Leeuwen, P. W. N. M.; Chadwick, J. C. Homogeneous Catalysts: activity, stability deactivation; Wiley; pp 11-23.

(67) Hakkinen, H. The Gold-sulfur Interface at the Nanoscale. Nat. Chem. 2012, 4, 443-455.

(68) Jover, J.; Garcia-Rates, M.; López, N. The Interplay between Homogeneous and Heterogeneous Phases of PdAu Catalysts for the Oxidation of Alcohols. ACS Catal. 2016, 6, 4135-4143. 